\title{
marges Marges
}

revue d'art contemporain Revue d'art contemporain

$04 \mid 2005$

Interdisciplinarité

\section{Le retournement transcréateur}

The trans-creative turn

\section{Rachida Triki}

\section{OpenEdition}

Journals

Édition électronique

URL : http://journals.openedition.org/marges/734

DOI : $10.4000 /$ marges.734

ISSN : 2416-8742

\section{Éditeur}

Presses universitaires de Vincennes

\section{Édition imprimée}

Date de publication : 15 octobre 2005

Pagination : 84-93

ISBN : 978-2-84292-248-1

ISSN : 1767-7114

Référence électronique

Rachida Triki, «Le retournement transcréateur », Marges [En ligne], 04 | 2005, mis en ligne le 15 octobre 2006, consulté le 07 mai 2019. URL : http://journals.openedition.org/marges/734 ; DOI : $10.4000 /$ marges.734

Ce document a été généré automatiquement le 7 mai 2019.

(c) Presses universitaires de Vincennes 


\section{Le retournement transcréateur}

The trans-creative turn

\section{Rachida Triki}

Abderrazak Sahli, sans titre

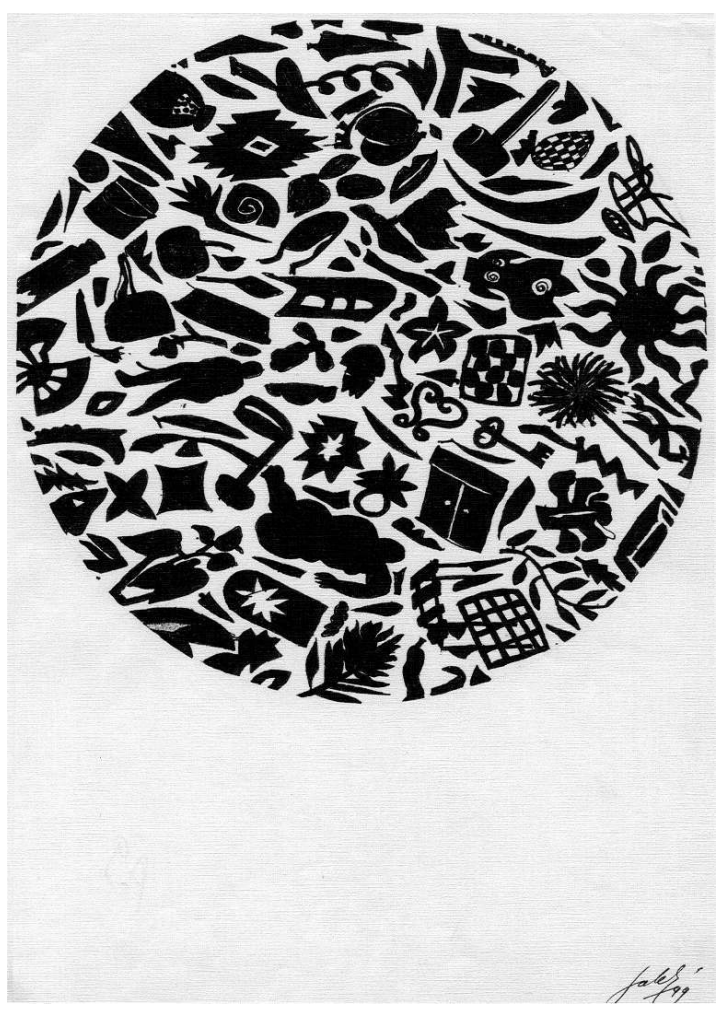

Hammamet 2002, acrylique sur papier, format 21/29

Réfléchir aux mutations, voire aux conversions qui peuvent se produire dans un parcours d'artiste, c'est interroger à la fois la nature du rapport que cet artiste entretient avec sa création, c'est-à-dire l'idée qu'il se fait de son œuvre, et le degré de mutabilité qui lui permettra ou non de continuer autrement. 
2 On pourrait partir de la définition que donne René Huyghe de l'œuvre d'art, à savoir une création consubstantielle au monde intérieur de l'artiste et à son monde extérieur. Il affirme en l'occurrence que « si réaliste que se croie le peintre [...] c'est son essence même qu'il révèle, qu'il avoue, par la manière dont il aborde et choisit, dont il transcrit la réalité ${ }^{1}$ » matérielle de son monde.

3 Ce qui nous intéresse ici, c'est le sentiment de cette consubstantialité et même, pourraiton dire, de cette transubstantialité qui fait passer l'artiste dans son œuvre en manifestant le lien entre l'art et sa propre vie et entre l'art et la vie, lien prôné aussi, en ce cas, par nombre de manifestes d'artistes au début du $20^{\mathrm{e}}$ siècle.

4 De manière générale, lorsque le sentiment de s'impliquer dans sa production est vécu comme un engagement dont l'issue a aussi une portée existentielle, cela suppose un minimum de conviction et le courage de risquer l'aventure. En ce sens, créer c'est résister, pour reprendre la formule de Gilles Deleuze; c'est oser un devenir qui est toujours une aventure : « Quelle terreur hante la tête de Van Gogh, prise dans un devenir tournesol? Il y faut les couleurs des peintres pour s'élever des perceptions vécues au percept, des affections à l'affect ${ }^{2} »$; en cela l'artiste est « un voyant, un devenant qui tente un combat incertain ${ }^{3} »$.

\section{Nécessité d'un processus créateur}

5 S'engager à créer autrement, fidèle à ses sensations que l'on rend visibles en osant d'autres procédés plus adéquats à sa nécessité intérieure et à sa vision singulière, c'est aussi résister à l'identification à un style pour être dans la vérité de son désir d'art. Picasso ne confiait-il pas à Hélène Parmelin qu'il ne pouvait pas faire autre chose que ce qu'il faisait, signifiant par là une sorte de nécessité qui le liait à ses créations. Aussi, n'estce pas un hasard de rencontrer souvent dans des propos de peintres les notions de vérité, de sincérité et de nécessité intérieure pour manifester une sorte d'être à sa création, un engagement total où n'interviendrait pas de considération extra-artistique. Cet accord avec soi-même, avec ses désirs, avec son besoin de produire ou tout simplement de s'exprimer à travers un médium semble décisif dans le processus créateur. C'est cette part de ce qu'on pourrait appeler sincérité ou constance à soi qui permet à la fois de résister face aux obstacles à la création, mais aussi de pouvoir renoncer à ce qui ne satisfait plus, d'être ouvert à d'autres modes de création et de pouvoir tout reprendre quand on en sent la nécessité.

6 Lorsque le travail artistique revêt cette dimension qu'on pourrait dire existentielle, place est faite au doute et à son intensification dans des périodes de crise. En effet, la conscience de ses limites et du danger de l'habitude quant aux modes de faire et aux modes tout court, peut venir d'un sentiment d'inadéquation à sa vision des choses ou à son for intérieur. Elle peut provoquer une insatisfaction telle qu'elle débouche sur un abandon ou un retournement radical. De fait, ce sentiment de divorce avec sa production peut surgir de manière plus aiguë chez les peintres contemporains dont l'environnement est assailli par d'innombrables reproductions, par un éclatement des genres, par une surcharge de styles et par les rapides désignations que nécessite la culture marchande.

7 Déjà, dans le premier manifeste des Nouveaux réalistes, Pierre Restany déclarait que « nous assistons aujourd'hui à l'épuisement et à la sclérose de tous les vocabulaires établis, [...] l'homme, s'il parvient à se réintégrer au réel, l'identifie à sa propre transcendance, qui est 
émotion, sentiment et finalement poésie, encore ${ }^{4} »$. Il donne l'exemple de Tinguely, d'Arman et de Spoerri.

Ce constat propre à la naissance de ce qui sera un mouvement, ne traduit pas uniquement l'engagement des nouveaux réalistes qui ont tenté de rejoindre un certain réel, par delà les labels de représentation du monde ou par delà les styles et les modes d'identification des différentes formes plastiques. On peut, tout à fait, l'élargir au questionnement des artistes qui reconnaissent d'autant plus l'épuisement des catégories et des normes instituées, en matière d'art, qu'ils sont conscients eux-mêmes de l'habitus qui presse à étiqueter le moindre tracé pour en étouffer la singularité et pour dissiper toute ambiguïté.

Il leur semble que le déjà vu et le trop vu ont fini non seulement par recouvrir entièrement le champ de réception mais aussi par occuper le geste de peindre au point de congédier toute forme de sensibilité et de rapport authentique avec le monde. Cette attitude n'est certes pas le lieu d'un raisonnement ou d'un froid diagnostic comparable au constat du critique d'art, mais d'abord l'expression d'un malaise éprouvé dans le sentiment de la vanité du faire.

\section{Désappropriation et rupture}

10 Le malaise ne vient pas uniquement du poids de la culture picturale qui prend à son piège toute nouvelle forme et qui occupe la mémoire visuelle et le geste créateur. Il est l'expression d'une situation où l'artiste se découvre en porte à faux avec son propre vécu et avec les sensations du monde. C'est donc un sentiment de désappropriation et de rupture qui peut dans son intensité provoquer la suspension de l'activité créatrice, mais il peut aussi être l'occasion de ce que j'appelle un « retournement transcréateur » qui puise de cette crise relationnelle des potentialités novatrices.

11 C'est le cas notamment de peintres qui prennent conscience des limites de leur démarche et de leur expression et choisissent d'autres modes de faire ou d'autres matériaux dans lesquels ils font passer leur sensation d'une manière qu'ils pensent plus appropriée à leur conviction. Quelle que soit l'intensité du retournement opéré dans le procédé choisi, lorsqu'il relève d'un désir de constance à soi, à savoir de cette vérité de l'art dont il était question plus haut, la conversion devient force de création, source de possibles. C'est en ce sens que l'artiste peut survivre à tout ce qui menace son autonomie, y compris au confort de sa reconnaissance qui est une autre forme d'aliénation.

La conversion peut alors ne pas constituer une rupture radicale qui priverait l'artiste de ses potentialités en le jetant dans l'altérité absolue d'un faire différent. Elle peut s'entendre comme mouvement de transcréation où le travail créateur s'inscrit dans un devenir polysensoriel. Il s'agit, à chaque fois, de se réapproprier un matériau pour le rendre autrement expressif selon les techniques qui conviennent. Plus généralement dans le domaine de la création artistique, on ne quitte pas un genre pour un autre, mais on fait passer différemment « le matériau dans la sensation ${ }^{5}$ » selon l'expression deleuzienne, en exploitant ses capacités d'expression.

13 Ce mouvement transcréateur est rendu possible par la mutabilité dans la constance à soi de l'artiste qu'on pourrait appeler résistance ou encore lyrisme, au sens où Maurice Merleau-Ponty parle du lyrisme de Paul Cézanne qui est celui de "l'existence toujours recommencée ${ }^{6} »$. Cette dimension de mutabilité est revendiquée par des artistes 
contemporains qui la vivent comme une nécessité. Robert Rauschenberg déclare qu'un peintre doit être "un chercheur, un inventeur perpétuel» et si Picasso récusait la qualification de chercheur et l'idée d'évolution d'un artiste, il n'en revendique pas moins le droit au changement parce que, comme il le dit, «les motifs différents exigent des modes d'expression différents »; c'est « une adaptation de l'idée qu'on veut exprimer et des moyens d'exprimer cette idée ${ }^{7}$ ».

Le retournement devient transcréateur quand l'artiste transgresse les limites et les obstacles qui l'enfermaient dans sa pratique pour re-dynamiser sa conduite créatrice dans un matériau différent par des figures autres, capables de capter de nouvelles forces (couleur, tracé, vitesse) qui sont autant de sensations où s'expriment plus intensément des affects.

La transcréation consiste aussi dans la capacité de passer d'une technique à une autre, d'une démarche à une autre dans un devenir-sujet de l'artiste qui exclut l'idée d'évolution ou de progrès. Peut-être qu'au regard du public, ce phénomène de conversion ne constitue-t-il plus, de nos jours, un phénomène remarquable dans la vie d'un artiste comme cela aurait été le cas dans des périodes antérieures. Certains pensent même que c'est un phénomène propre à l'art contemporain que de ne pas tenir à un seul genre.

Nathalie Heinich situe ce positionnement dans la désignation des artistes par le terme de plasticiens $^{8}$, qui s'est imposé pour unifier plusieurs approches susceptibles d'être adoptées par la même personne comme la peinture, la sculpture et l'architecture comprises, par exemple, dans la pratique du happening ou des installations. Mais elle reconnaît aussi qu'il y a là un enjeu qui est celui de l'identification de l'artiste à travers les preuves d'une authenticité de l'intention du créateur pris dans sa singularité. C'est en dernière instance, cette authenticité qui garantit la crédibilité des retournements de l'artiste. Le cas de figure de l'artiste peintre tunisien Abderrazak Sahli est à ce propos intéressant.

\section{Le retournement transcréateur d'Abderrazak Sahli}

17 Né en 1941 à Hammamet, Sahli a fait des études à l'École des beaux-arts de Tunis, spécialité peinture (1969) puis à l'Université de Paris 8 en Arts plastiques et à l'École des beaux-arts de Paris, spécialité gravure (1974). Il a séjourné en France jusqu'en $1987^{10}$. Entre 1965 et 1987, il adopte une démarche qui se manifeste dans deux périodes. De 1965 à 1975, il travaille à un jeu de modifications et de déplacements de la calligraphie arabe traitée librement dans une configuration qui rappelle en les déterritorialisant les papiers officiels et les parchemins qui font autorité. En fait, comme il le dit, déjà lors de sa formation coranique à la Zitouna, puis de son passage à l'École des beaux-arts de Tunis, il a ressenti la dimension problématique du dit et du graphique. Cela a donné lieu à ce qu'il appelle un " combat avec les mots, un travail acharné sur les mots ». Contre sa difficulté à prononcer (légère dyslexie), contre l'institué et l'assimilation d'une écriture normée dont l'aboutissement est le discours officiel, il oppose une pseudo-écriture par les moyens plastiques que permettent les signes de la calligraphie arabe. Dans un jeu hors sens de tracés cursifs et déliés, il inscrit sous forme de papiers officiels avec tampon et paraphe, des textes illisibles qui portent en dérision l'autorité de l'écrit. Il fait aussi des performances et de la poésie sonore où les mots passent à la vocalise dans le même hors sens mais dont la sensorialité du rythme et du souffle rappelle les transes des soufis. 

d'inversion, de détournement et de camouflage du verbe s'élargit à l'icône et se manifeste dans une série de portraits-photos sur toile en bas desquels il inscrit l'illisibilité d'un graphisme ni vraiment latin, ni vraiment arabe. La poïétique de la disparition opère au niveau des portraits un effacement des yeux qui annule la cartographie du visage. La défiguration est amplifiée par des tracés couleurs qui traversent peintures, photos et parchemins en annulant leur identité dans la même opération. Le travail de transfiguration se déplace sur le corps propre et le monde. Sahli fait de son corps un lieu culturel d'arabe émigré. Sa main peinte devient langue d'exil dans des performances où la gestuelle et la vocalise illimitent le corps propre. art, le place à la fois devant la difficulté matérielle de peindre avec de modestes moyens, dans un environnement pictural dominé par l'abstraction géométrique ou lyrique et par une semi-figuration onirique dans lesquels il ne se reconnaît pas.

se retire dans une ancienne maison à Hammamet dont il fait son atelier et adopte une démarche nouvelle où il fait passer sa sensation du lieu et du milieu dans un traitement proche du mouvement de la Figuration libre. Cela lui permet à la fois un éclatement des formes et leur simplification qui porte la peinture à la limite de sa poésie sonore - ce sera l'occasion d'ailleurs de certains happenings associant peinture et poésie. Ce retournement est transcréateur au sens où il permet une meilleure expression du rapport du peintre à son monde. Ce processus va se développer pour devenir son quotidien puisque Sahli va aussi puiser dans sa rencontre avec les techniques de Claude Viallat (Support-surface), la répétition des modules, le support sans cadre, le colorisme, la saturation des toiles et surtout l'ouverture à des matériaux tels que le drap usé, la toile de jute et de vieux vêtements dans lesquels il fait passer son monde de sensations. Il transgressera la modestie des moyens pour les élever à la transcendance. Il travaillera avec l'objet trivial, le Kortass (série de 1989), modeste paquet en papier kraft de l'épicier du coin, objet banal qui contient et qui cache pour en exhiber les possibles. Cet objet devient structure, volume, support et même élément sonore dans les performances vocales. D'autres éléments comme les grandes toiles servant à la cueillette d'olives ou le sèche-linge en bois qu'on met sur le brasier sont « désustentialisés » et " énergétisés » dans un univers plastique polychrome. Dans le même temps, la poésie sonore de Sahli intègre des happenings dans une mise en scène où sont convoqués des objets symboliques à travers un jeu de métissage entre art et sacré.

21 Il aura fallu le retour dans sa ville natale et des aléas pour que se fasse cette conversion opérée par contagion des mouvements comme Support-surface, Nouvelle figuration ou même Arte povera que Sahli aura connus en France, loin de chez lui. Toutes ces rencontres ont donné l'occasion à sa démarche de continuer à créer en réintégrant son corps, sa voix, son rythme et son milieu. C'est probablement cette forme d'authenticité singulière qui permet d'exister comme artiste à travers toutes les mutations possibles. 
Abderrazak Sahli, sans titre

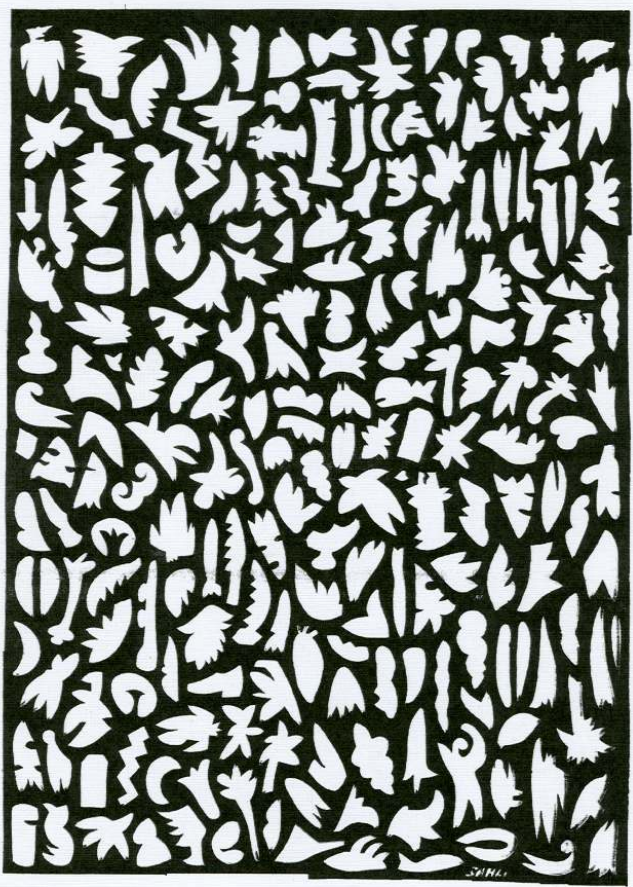

Hammamet 2002, acrylique sur papier, format 21/29.

Abderrazak Sahli, sans titre

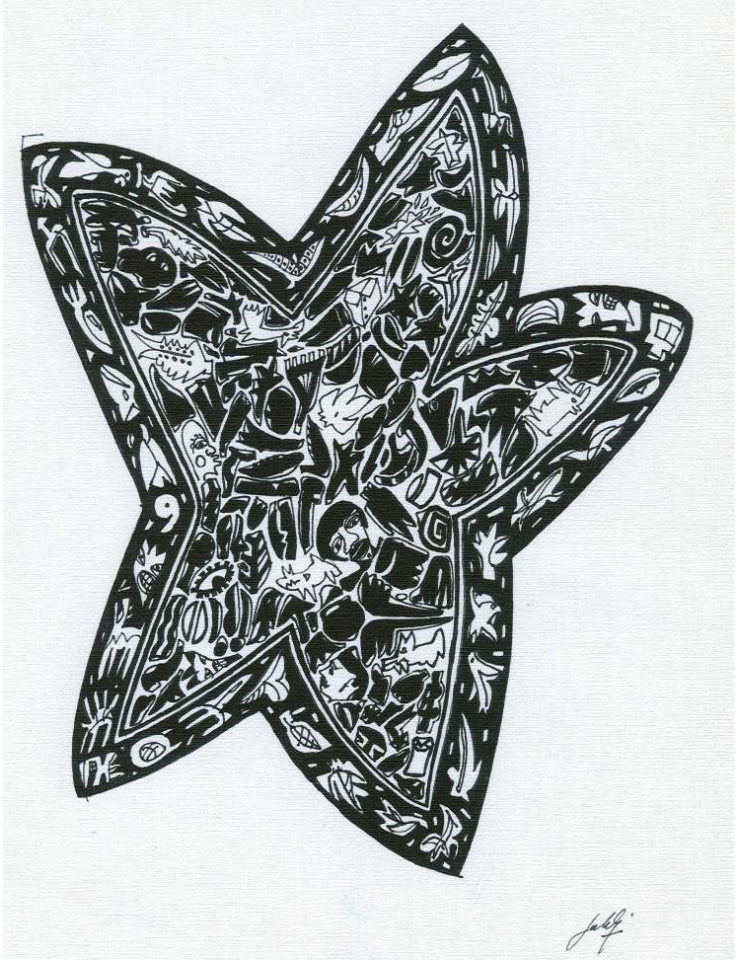

Hammamet 2002, acrylique sur papier, format 21/29. 
Abderrazak Sahli, sans titre (détail)

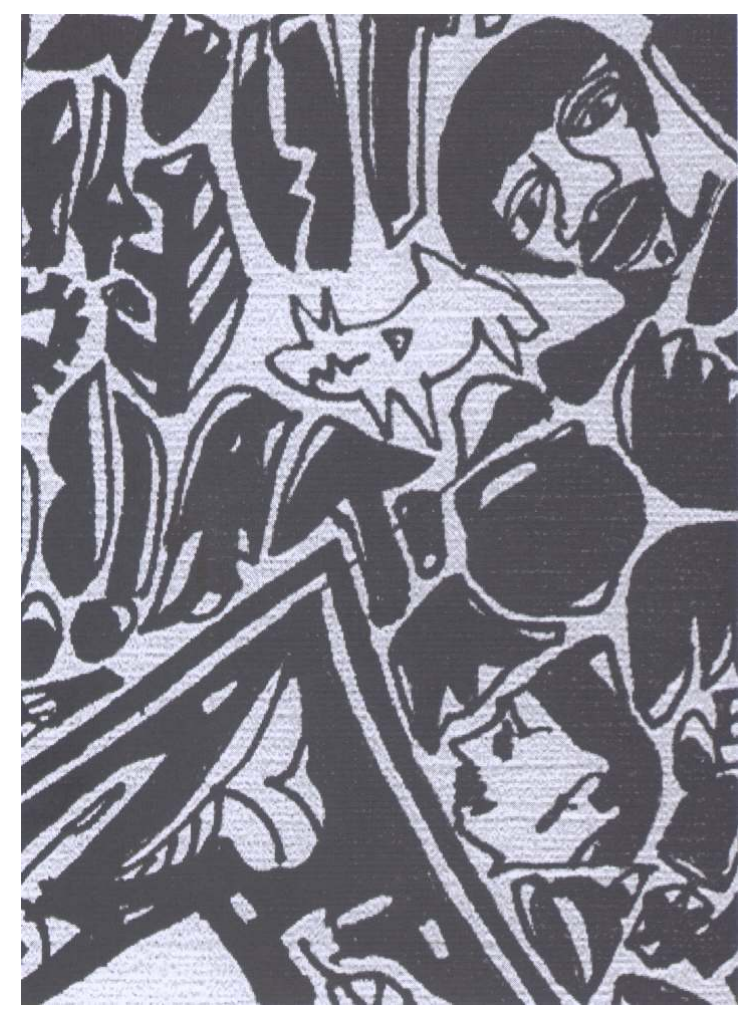

Hammamet 2002, acrylique sur papier, format 21/29.

\section{NOTES}

1. René Huyghe, Dialogue avec le visible, Paris, Flammarion, 1955, p. 105.

2. Gilles Deleuze, Félix Guattari, Qu'est-ce que la philosophie ?, Paris, Minuit, 1991, p. 106.

3. ibid., p. 160-161.

4. «Premier manifeste du 16 avril 1960 à Milan», dans Les Nouveaux Réalistes, Paris, éditions Planète, 1968.

5. Gilles Deleuze, Félix Guattari, op. cit., p. 157.

6. Maurice Merleau-Ponty, Sens et non sens, Paris, Nagel, 1948, p. 30.

7. Pablo Picasso, Propos sur l'art, Paris, Gallimard, Paris, 1998, p. 19.

8. Nathalie Heinich, Le Triple jeu de l'art contemporain, Paris, Minuit, 1998, p. 99.

9. ibid., p. 130-131.

10. Sahli a participé à la Biennale de Paris de 1971, a exposé à la Galerie Arlogos de Nantes en 1980, à la Galerie Créatis à Paris en 1980. Il a participé à l'exposition «Livres d'artistes, livres objets » en 1982 au Centre Georges Pompidou à Paris. 


\section{RÉSUMÉS}

Chaque vie d'artiste est ponctuée de rencontres et de bouleversements souvent capitaux. L'artiste tunisien Abderrazak Sahli a su à chaque instant, questionner sa pratique pour insuffler une nouveauté dans ses peintures, collages ou happenings. C'est alors un singulier « retournement transcréateur » qui s'opère chez celui qui poursuit sa quête de l'authenticité.

The life of an artist is punctuated with meetings and often crucial overthrows. The Tunisian artist Abderrazak Sahli has constantly known how to question his own practice in order to bring about a new breath to his paintings, collages or happenings. A specific "trans-creative turn" happens then with someone who pursues a quest for authenticity.

\section{AUTEUR}

\section{RACHIDA TRIKI}

Professeure en Philosophie, Université de Tunis.

Présidente de l'Association Tunisienne D’Esthétique et de Poḯtique. 